\title{
Effect of the strain Bacillus amyloliquefaciens FZB42 on the microbial community in the rhizosphere of lettuce under field conditions analyzed by whole metagenome sequencing
}

\author{
Magdalena Kröber ${ }^{1}$, Daniel Wibberg ${ }^{1}$, Rita Grosch ${ }^{2}$, Felix Eikmeyer ${ }^{1}$, Bart Verwaaijen ${ }^{1}$, \\ Soumitra P. Chowdhury ${ }^{3}$, Anton Hartmann ${ }^{3}$, Alfred Pühler ${ }^{1}$ and Andreas Schlüter ${ }^{1 *}$ \\ ${ }^{1}$ Center for Biotechnology, Institute for Genome Research and Systems Biology, Bielefeld University, Bielefeld, Germany \\ ${ }^{2}$ Leibniz-Institute of Vegetable and Ornamental Crops, Großbeeren, Germany \\ ${ }^{3}$ Research Unit Microbe-Plant Interactions, Helmholtz Zentrum München, German Research Center for Environmental Health (GmbH), Neuherberg, Germany
}

\section{Edited by:}

Michael Schloter, Helmholtz

Zentrum München, Germany

Reviewed by:

Leo Van Overbeek, W-UR Plant

Research International, Netherlands

David John Studholme, University of

Exeter, UK

Anja Bettina Dohrmann, Johann

Heinrich von Thünen Institute,

Germany

*Correspondence:

Andreas Schlüter, Center for Biotechnology, Institute for Genome

Research and Systems Biology,

Universität Bielefeld, Universitätsstr.

27, 33615 Bielefeld, Germany

e-mail:aschluet@

cebitec.uni-bielefeld.de
Application of the plant associated bacterium Bacillus amyloliquefaciens FZB42 on lettuce (Lactuca sativa) confirmed its capability to promote plant growth and health by reducing disease severity (DS) caused by the phytopathogenic fungus Rhizoctonia solani. Therefore this strain is commercially applied as an eco-friendly plant protective agent. It is able to produce cyclic lipopeptides (CLP) and polyketides featuring antifungal and antibacterial properties. Production of these secondary metabolites led to the question of a possible impact of strain FZB42 on the composition of microbial rhizosphere communities after its application. Rating of DS and lettuce growth during a field trial confirmed the positive impact of strain FZB42 on the health of the host plant. To verify B. amyloliquefaciens as an environmentally compatible plant protective agent, its effect on the indigenous rhizosphere community was analyzed by metagenome sequencing. Rhizosphere microbial communities of lettuce treated with $B$. amyloliquefaciens FZB42 and non-treated plants were profiled by high-throughput metagenome sequencing of whole community DNA. Fragment recruitments of metagenome sequence reads on the genome sequence of B. amyloliquefaciens FZB42 proved the presence of the strain in the rhizosphere over 5 weeks of the field trial. Comparison of taxonomic community profiles only revealed marginal changes after application of strain FZB42. The orders Burkholderiales, Actinomycetales and Rhizobiales were most abundant in all samples. Depending on plant age a general shift within the composition of the microbial communities that was independent of the application of strain FZB42 was observed. In addition to the taxonomic profiling, functional analysis of annotated sequences revealed no major differences between samples regarding application of the inoculant strain.

Keywords: metagenome sequencing, taxonomic profiling, B. amyloliquefaciens FZB42, lettuce, rhizosphere, fragment recruitment

\section{INTRODUCTION}

Phytopathogenic organisms are a serious threat to food production and storage affecting crop plants and vegetables. Crop losses of approximately $20 \%$ and more were caused by plant pathogens worldwide depending on the particular crop (Oerke, 2006). Crop rotation, breeding of resistant cultivars and the use of chemical pesticides are the preferred approaches to reduce the effects of phytopathogens. However, these strategies are insufficient, especially to control root diseases caused by soilborne plant pathogens of economically important crops (Johri et al., 2003). Additionally, intensive crop management practices increase the problems concerning accumulation of pathogens in the soil (Chellemi, 2002). The facts that excessive use of chemical pesticides caused several environmental problems like soil pollution (Asaka and Shoda, 1996) and that society is becoming more conscious about the environment support the development of alternative control strategies worldwide (Leistra and Matser, 2004; Wang et al., 2004; Alabouvette et al., 2006). Currently, it is well documented that the treatment of plants with plantassociated microorganisms can be effective to decrease the negative impact brought about by infections of the plants by pests (Kazempour, 2004; Scherwinski et al., 2008; Andrews et al., 2012).

Several products based on the application of plant-associated bacteria such as Bacillus spp. are commercially available (Choudhary and Johri, 2009). The ability of members of the Gram-positive genus Bacillus to form spores is advantageous for preparation of bacterial formulations, storage and product shelf life (Tiago et al., 2004). The effectiveness of these strains relies on different mechanisms. Efficient strains colonize the root system of 
the host plant, promote plant growth and are able to activate their defense system. This phenomenon of triggering the systemic resistance reaction of the plant has been termed rhizobacteria-induced systemic resistance (ISR) (Arguelles-Arias et al., 2009). Another mechanism that contributes to plant protection is the secretion of different secondary metabolites with antibacterial or antifungal impact on phytopathogenic organisms. In addition, the competition for nutrients and especially iron between biocontrol strains and pathogens is an important factor in the protection of the plant (Arguelles-Arias et al., 2009).

Bacillus amyloliquefaciens FZB42 is known for its plant growth promoting properties and its ability to suppress different plant pathogens (Grosch et al., 1999; Idriss et al., 2004; Koumoutsi et al., 2007). Pot and field experiments demonstrated that the strain FZB42 is able to effectively colonize the rhizosphere of lettuce during host plant cultivation and promotes significant suppression of bottom rot disease caused by Rhizoctonia solani (Chowdhury et al., 2013). High rhizosphere competence referring to the survival and rhizosphere colonization potential (Bulgarelli et al., 2013) is a key factor for a successful improvement of plant traits and control of plant pathogens by plant-protective agents (Lugtenberg and Kamilova, 2009). Although the commercially available strain FZB42 is a beneficial bacterium, its application may have a negative impact on the indigenous microbial rhizosphere community and associated important ecological functions (Winding et al., 2004). Genome analyses of B. amyloliquefaciens revealed that the organism harbors eight different gene clusters comprising genes associated with the production of secondary metabolites providing it with its biocontrol properties (Chen et al., 2007). These secondary metabolites can be classified into three different categories: the polyketides macrolactin $(m l n)$, bacillaene $(b a e)$, and difficidin $(d f n)$ featuring antibacterial properties (Chen et al., 2006), the cyclic lipopeptides surfactin $(s r f)$, fengycin $(f e n)$, and bacillomycin (bmy) providing mainly antifungal properties (Koumoutsi et al., 2004) and the third category including the iron-siderophore bacillibactin (bac) and a putative siderophore the synthesis of which is encoded by the nrs operon.

Due to the high potential of producing secondary metabolites and their possible impact on other microorganisms within the microbial community in the rhizosphere of host plants, the complex interaction between an inoculant strain and the indigenous microbial rhizosphere community has to be analyzed. Knowledge regarding the microbial ecology of the target habitat "rhizosphere" is required for reasonable risk assessment studies related to the application of beneficial plant associated bacterial strains.

To increase the knowledge about the interaction of the strain FZB42 with indigenous rhizosphere community members of lettuce, field trials were carried out at natural occurring pathogen pressure in the field and at higher pathogen pressure achieved by inoculation of the bottom rot pathogen $R$. solani at the same field site (Chowdhury et al., 2013). During the growth period of lettuce whole community DNA samples were isolated from the rhizospheres of lettuce that either were treated with the strain FZB42 (1 week before planting and 4 days after planting) or not treated. Samples from both experiments were collected at planting, 2 weeks and 5 weeks after planting. Analysis of DNA samples from these field trials after 2 and 5 weeks of cultivation of the host plants by $16 \mathrm{~S}$ rRNA gene amplicon terminal restriction fragment length polymorphism (T-RFLP) revealed no major impact on the general composition of the microbial community regarding the application of the inoculant strain. However, a temporal shift independent from the application of strain FZB42 was observed (Chowdhury et al., 2013).

In contrast to T-RFLP analyses, metagenome studies by means of whole community DNA extraction, high-throughput sequencing and various kinds of sequence analyses applying bioinformatic tools are exceptionally convenient strategies to provide an extended insight into the whole microbial community within a habitat e.g., the rhizosphere. MG-RAST is an established platform to analyze and compare metagenome sequence data in several approaches (Meyer et al., 2008). Within MG-RAST, taxonomic profiling of the community based on metagenome sequences is performed using the NCBI GenBank database comprising sequences of more than 380,000 species. It therefore provides an exceptionally suitable basis for the annotation of metagenome sequences (Benson et al., 2011). Additionally to taxonomic classifications, the MG-RAST software platform provides the possibility to compare functional subsystems based on the SEED resource (http://www.theSEED.org), (Aziz et al., 2008; Overbeek et al., 2014).

To obtain deeper and more refined insights into microbial rhizosphere communities of lettuce, the same DNA samples as for the T-RFLP analysis described above (Chowdhury et al., 2013) were used in a comparative whole metagenome sequencing approach to follow up changes in the taxonomic community profiles during the growth period of lettuce and to identify dominant rhizosphere microorganisms. In addition to the samples used in the 16S rRNA T-RFLP analysis, DNA samples isolated from the rhizosphere at planting 1 week after the first application of strain FZB42 were analyzed by metagenome sequencing in order to reveal possible shifts in the microbial community within the first 2 weeks of cultivation in the field. Previous field trials showed that during this time infections with the phytopathogen $R$. solani are most likely to occur (Grosch et al., 2004).

Another objective of this study was to elucidate the competitiveness and survivability of the inoculant strain FZB42 within the bacterial community in the course of host plant cultivation. The ability of B. amyloliquefaciens FZB42 to successfully colonize the rhizosphere is an assumption for reducing disease severity (DS) caused by phytopathogens (Lugtenberg and Kamilova, 2009; Barret et al., 2011; Ghirardi et al., 2012). Expression of genes conferring antifungal activity often is regulated in a cell-density dependent manner (Steidle et al., 2002) and hence corresponding microorganisms should be highly competent in rhizosphere colonization to exert antifungal activity. Proving the establishment of this strain in the rhizosphere of lettuce therefore is the prerequisite for the following analyses addressing alterations in the community profiles between with FZB42 treated and non-treated samples. Therefore, fragment recruitments were performed in which metagenome sequence reads were mapped to the known genome sequence of $B$. amyloliquefaciens FZB42 under strict 
settings in order to track the occurrence of this strain in the rhizosphere.

In addition to taxonomic profiling, whole metagenome sequencing also allows functional characterization of the rhizosphere community. Studies of microbial rhizosphere communities of different plant species showed that their roots are colonized by only a few dominating phyla (Bulgarelli et al., 2013; Chaparro et al., 2013). These show a common set of metabolic functions connected to genes involved in carbohydrate, nitrogen and amino acid metabolism. This observation has led to the assumption that specific functions are essential for a successful colonization of this habitat during the growth of the plants. The treatment of plants with B. amyloliquefaciens FZB42 and its secretion of secondary metabolites with antimicrobial properties potentially may lead to shifts within rhizosphere microbial functional subsystems affecting e.g., availability of nutrients for the plant and other important properties in the rhizosphere. Furthermore, the functional subsystem of virulence and defense mechanisms was analyzed since alterations within this category may be caused by application of the inoculant strain FZB42.

\section{MATERIALS AND METHODS}

\section{FIELD TRIAL AND PREPARATION OF DNA SAMPLES}

A field trial was carried out at the Institute of Vegetable and Ornamental Crops (Golzow, Germany, 52 $34^{\prime} \mathrm{N}, 14^{\circ} 30^{\prime} \mathrm{E}$ ) to determine the ability of $B$. amyloliquefaciens FZB42 to colonize the rhizosphere of lettuce plants (cv. Tizian, Syngenta, Bad Salzuflen, Germany) and the potential impact of the treatment with this strain on the composition of the microbial community within this habitat. Prior to the cultivation in the field the young plants were grown in peat blocks at $20 / 15^{\circ} \mathrm{C}(16 / 8 \mathrm{~h}$, day/night cycle) and were then transferred in the 3-4 leaf state to the field with alluvial loam (total N 112; P 32.3; K 17.4; and $\mathrm{Mg}$ $9.1 \mathrm{mg} / 100 \mathrm{~g}$ soil; $\mathrm{pH} 6.5$ ). Lettuce plants were first treated with FZB42 in the 2-3-leaf stage 1 week before planting into the field beds. Each seedling tray with 150 plants was watered with 1.741 spore suspension $\left(10^{7} \mathrm{CFU} / \mathrm{ml}\right)$ of FZB42. Four days after planting, the lettuce plants at the 3-4-leaf stage were treated with a spore suspension $\left(10^{7} \mathrm{CFU} / \mathrm{ml}\right)$ of FZB42 for a second time. An amount of 0.51 spore suspension was applied by hand sprayer to each bed with the size of $6.75 \mathrm{~m}^{2}$ with 11 plants per $\mathrm{m}^{2}$. The experiment comprised non-treated (control) and treated (inoculated) plants at planting ( $\mathrm{t} 0), 2$ weeks ( $\mathrm{t} 1$ ) and 5 weeks ( $\mathrm{t} 2$ ) after planting of lettuce into the field. Total community DNAs were isolated from the rhizosphere of three lettuce plants per replicate and combined prior to DNA isolation. Samples were taken from four replicates randomly arranged in the experimental field. This approach resulted in the analysis of 12 plants per treatment and time point. The experimental design and sampling regime of this study is outlined in detail in a previous publication (Chowdhury et al., 2013).

\section{HIGH-THROUGHPUT SEQUENCING OF METAGENOMIC DNA}

Whole metagenome shotgun libraries were constructed based on the metagenomic DNA preparations extracted from the rhizosphere of lettuce. Prior to library preparation $3.3 \mathrm{ng}$ DNA from three replicates (six plants each) were pooled per treatment and sampling time point. Accordingly, each metagenomic library comprises rhizosphere total community DNA preparations from 12 plants per treatment. Sequencing of these libraries was performed on the MiSeq system (Illumina) according to protocols provided by Illumina.

\section{BIOINFORMATIC ANALYSIS OF METAGENOME SEOUENCE DATA}

Metagenome reads were processed and analyzed within the MG-RAST software package (Meyer et al., 2008; Wilke et al., 2013). Sequence data are available from the MG-RAST platform under the following IDs: t0 control: http://meta genomics.anl.gov/linkin.cgi?metagenome $=4535147.3$, t0 inoculated: http://metagenomics.anl.gov/linkin.cgi?metagenome $=4535$ 148.3, t1 control: http://metagenomics.anl.gov/linkin.cgi?meta genome $=4535149.3$, t1 inoculated http://metagenomics.anl.gov/ linkin.cgi? metagenome $=4535150.3$, t2 control: http://meta genomics.anl.gov/linkin.cgi?metagenome $=4535151.3$, t2 inoculated: http://metagenomics.anl.gov/linkin.cgi?metagenome $=$ 4535152.3 .

Taxonomic profiling and determination of $\alpha$-diversity was performed within MG-RAST applying the "Reprehensive Hit Classification" option using the GenBank database as reference with the following settings: maximum e-value cut off of $1^{*} 10^{-5}$, minimum identity of $80 \%$, and minimum alignment length of 15 measured in amino acids for proteins and base pairs for RNA databases. Taxonomic information was inferred from the best BLAST hit applying the "Representative Hit" option within MGRAST. Compositions of microbial communities were compared based on normalized ratios for each taxon identified within a specific dataset. Ratios of identified taxa were calculated by referring them to the total amount of classified sequences within each dataset. The deduced taxonomic profiles were visualized by means of Microsoft Excel (2008). Additionally, MA-plots were calculated. These plots visualize the distribution of annotated genera within control and inoculated sample sets by plotting the logarithmic ratios between the numbers of annotated sequences ( $M$-value) against the logarithmic mean average of annotated sequences ( $A$-value) of each genus.

\section{ANNOTATION OF FUNCTIONAL SUBSYSTEMS}

Analysis of the relevant functional subgroups was performed using the SEED subsystems technology within the MG-RAST software package with default settings. Proteins deduced from metagenome reads were compared to the FIGfam protein family collection within MG-RAST and then classified according to the predefined subsystems applying the SEED technology implemented in MG-RAST (Aziz et al., 2008; Meyer et al., 2008; Overbeek et al., 2014). All metagenome sequences matching a specific subsystem were counted and this value was then divided by the number of sequences that were assigned to any subsystem to give fractions and therefore normalized, comparable values (Meyer et al., 2008).

\section{MAPPING OF METAGENOME SEQUENCE READS TO THE B. AMYLOLIOUEFACIENS FZB42 GENOME}

Metagenome sequence reads of the six datasets were mapped to the B. amyloliquefaciens FZB42 reference genome (GenBank 
Accession: CP000560) by means of the gsMapper program (Roche Genome Analyzer Data Analysis Software Package, version 2.8) in order to follow the occurrence of the inoculant strain in the rhizosphere. Strict settings of the gsMapper (98\% sequence identity, minimum 225 bp length, minimum $90 \%$ overlap) were used for this fragment recruitment to specifically map reads originating from B. amyloliquefaciens FZB42. Multiple contigs and corresponding consensus sequences were generated from the mapped reads. To visualize the positions of the mapped reads in the B. amyloliquefaciens FZB42 genome, a Circos plot of the resulting contigs for each time point was calculated (Krzywinski et al., 2009). For the determination of the traceable amounts of strain FZB42 in the course of the cultivation, the normalized numbers of metagenome reads only matching specific $B$. amyloliquefaciens FZB42 genes were used.

\section{RESULTS \\ METAGENOME SEOUENCING OF LETTUCE RHIZOSPHERE MICROBIAL COMMUNITIES}

To determine the capability of B. amyloliquefaciens FZB42 to colonize the lettuce rhizosphere, taxonomic and functional profiles of microbial rhizosphere communities were deduced from corresponding metagenome sequence data. Since in the previous study (Chowdhury et al., 2013) comparison of T-RFLP patterns obtained for replicates of rhizosphere DNA preparations revealed consistent results over the replicates per sample, it was decided to pool the rhizosphere samples per treatment and sampling time point in the metagenome study described here. Table 1 shows the results of the metagenome sequencing approach of six different DNA-libraries from a field trial that schematically is represented in Figure 1.

Table 1 | Statistics of metagenome sequencing of DNA preparations from the rhizosphere of Lactuca sativa treated with B. amyloliquefaciens FZB42 (+FZB42) and corresponding controls.

\begin{tabular}{|c|c|c|c|c|c|c|}
\hline & to control & to inoculated & t1 control & t1 inoculated & t2 control & t2 inoculated \\
\hline High quality sequences & $1,869,056$ & $2,446,824$ & 783,195 & $1,470,887$ & $1,550,845$ & 971,074 \\
\hline Mean GC content $(\%)$ & $58 \pm 12$ & $58 \pm 11$ & $62 \pm 10$ & $62 \pm 10$ & $61 \pm 11$ & $60 \pm 12$ \\
\hline Annotated sequences & 624,429 & 706,259 & 169,574 & 286,817 & 344,059 & 203,447 \\
\hline
\end{tabular}

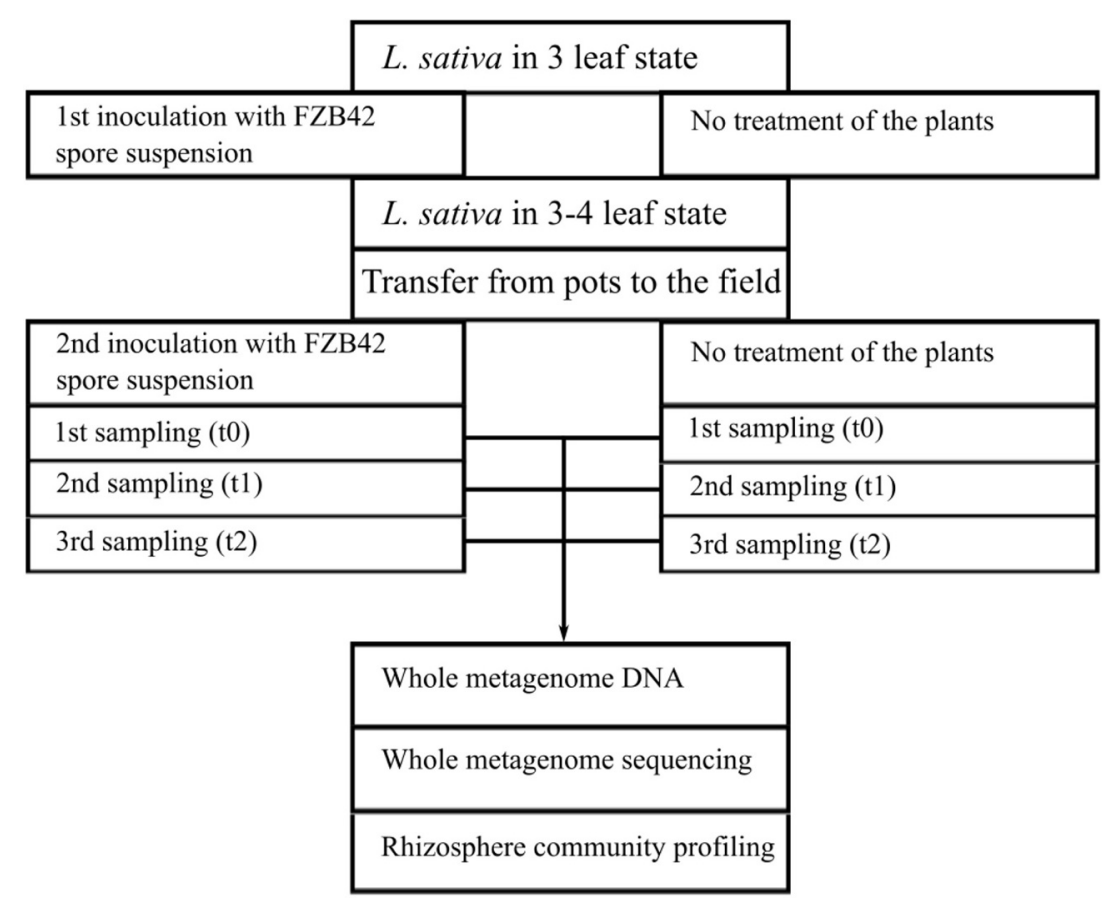

FIGURE 1 | Scheme of the lettuce field cultivation experiment. Lettuce was either inoculated with B. amyloliquefaciens FZB42 or not treated. Samples of both experiments were drawn at planting (t0), 2 weeks (t 1 ), and 5 weeks (t2) of cultivation from the rhizosphere of the inoculated and non-treated plants. Rhizosphere samples were used for whole community DNA extraction and subsequently for metagenome sequencing. Annotated metagenome reads were used for taxonomic and functional profiling of the microbial communities within the lettuce rhizospheres. 
Yields of the sequencing runs were determined to be between 783,195 and 2,446,824 reads after quality control (Table 1). Taxonomic annotation with the implemented "Representative Hit Classification" of MG-RAST against the GenBank database resulted in the annotation of between 169,574 and 706,259 sequence reads considering all taxonomic domains. Classified reads represent between 19.5 and $33.4 \%$ of all sequences. Among all annotated taxonomic domains $98.2 \%$ of the reads were assigned to the domain Bacteria. (Table 1).

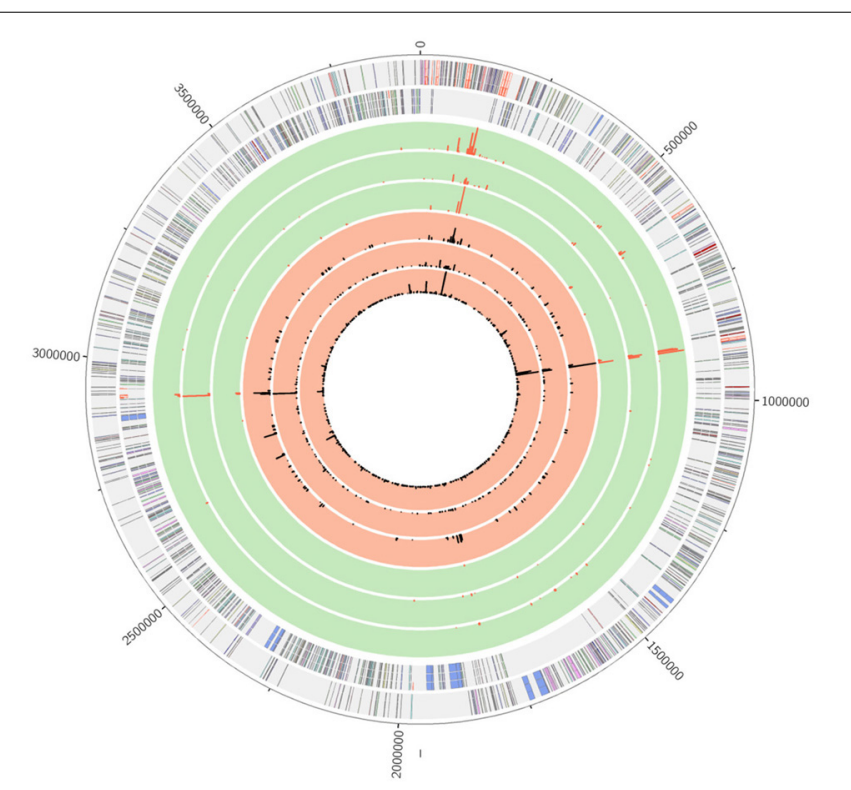

FIGURE 2 | Mapping of metagenome sequence reads to the B. amyloliquefaciens FZB42 genome. The circles represent from inner to outer most: (i) t0 inoculated sample; (ii) t1 inoculated sample; (iii) t2 inoculated sample; (iv) t0 control; (v) t1 control; (vi) t2 control; (vii) annotated coding sequences in both strands; (viii) scale in bp. The replication initiation gene $d n a A$ was chosen as first gene of the chromosome. Coding sequences are colorized depending on their gene function according to COG (Clusters of Orthologous Groups of proteins) categories. The scale for mapped reads represents the relative abundance of reads per base normalized to the maximum value of mapped reads per base.

\section{ESTIMATION OF LETTUCE RHIZOSPHERE COLONIZATION BY B. AMYLOLIQUEFACIENS FZB42 BASED ON FRAGMENT RECRUITMENT ANALYSES}

Prior to taxonomic profiling and comparison of community profiles, metagenome sequences were used to estimate rhizosphere colonization of the inoculant strain in order to relate potential changes within the taxonomic and functional profiles to the application of B. amyloliquefaciens FZB42. Mapping of the metagenome sequences to the reference genome of $B$. amyloliquefaciens FZB42 revealed that the strain is able to colonize the rhizosphere of lettuce over the course of the whole growth period (Figure 2 and Table 2).

Comparison of the amount of mapped reads and the resulting assembled contigs between samples taken at the different time points showed that for the first sample ( $\mathrm{t} 0)$ more reads from inoculated rhizospheres were mapped to the reference genome. Figure 2 clearly shows that within the first inoculated sample a wide set of genes of FZB42 is covered by 10,143 metagenome sequences adding up to a contig size of $203,115 \mathrm{bp}$. In the course of the experiment, the number of sequences specifically matching the B. amyloliquefaciens FZB42 genome decreased considerably to 5539 in the $t 1$ and 3478 in the $t 2$ inoculated sample, but still a part of the reference genome is covered by metagenome sequences. In contrast, metagenome sequence reads from non-inoculated samples mapping to the reference genome accumulate in very small sections of the genome.

In addition to the number of mapped reads to the reference genome, the corresponding annotated genes were determined. The number of FZB42 genes covered by metagenome reads was considerably higher in all inoculated samples compared to the control samples and are distributed over the whole genome (Table 2). Sequence reads from control samples that were mapped to the reference genome mainly represent genes encoding rRNAs and mobile genetic elements, which most probably are widely spread in species of the genus Bacillus. Among the completely covered genes for these samples, only 19 were already annotated and either corresponded to rRNA genes or other conserved housekeeping genes or have no assigned function yet. Accordingly, reads recruited to the $B$. amyloliquefaciens FZB42 genome from the metagenome data sets of the control samples most probably originate from indigenous Bacillus species that share particular genes

Table 2 | Mapping of reads, contigs and genes from the metagenome datasets to the $B$. amyloliquefaciens FZB42 reference genome.

\begin{tabular}{|c|c|c|c|c|c|c|}
\hline & to control & to inoculated & t1 control & t1 inoculated & t2 control & t2 inoculated \\
\hline Mapped reads ${ }^{a}$ & 7,859 & 10,143 & 2,808 & 5,539 & 5,251 & 3,478 \\
\hline Mapped reads $(\%)^{b}$ & 0.21 & 0.20 & 0.18 & 0.18 & 0.17 & 0.18 \\
\hline Number of contigs ${ }^{c}$ & 28 & 619 & 48 & 256 & 75 & 155 \\
\hline Contig size $[b p]^{d}$ & 5,957 & 203,115 & 9,038 & 80,095 & 13,421 & 44,859 \\
\hline Covered genes $^{\mathrm{e}}$ & 25 & 598 & 58 & 266 & 74 & 162 \\
\hline
\end{tabular}

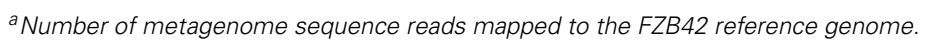

${ }^{b}$ Ratio of mapped reads within all high quality metagenome sequence reads (see Table 1).

${ }^{c}$ Number of contigs assembled from mapped reads.

${ }^{d}$ Resulting contig size of the assembled contigs.

e Number of genes hit by metagenome sequence reads. 
or DNA elements with B. amyloliquefaciens FZB42. However, 835 B. amyloliquefaciens FZB42 genes featured recruited metagenome reads originating only from the inoculated samples. These genes found no matching reads in any control sample. The 835 specific genes were used to trace occurrence of the inoculant strain during the growth period of lettuce. Metagenome sequence reads hitting any of these specific genes with high stringency (settings are given in Material and Methods) were counted for the inoculated samples for the sampling time points $\mathrm{t} 0, \mathrm{t} 1$, and $\mathrm{t} 2$ after normalization of datasets (Supplementary table 1). As mentioned above, none of the reads from the control samples matched any gene determined to be specific for B. amyloliquefaciens FZB42. It appeared that recruited read counts from with FZB42 treated samples decreased from $100(\mathrm{t} 0)$ to $64 \%(\mathrm{t} 1)$ to $55 \%$ at the end of the experiment (t2). In summary, fragment recruitments proved occurrence of the inoculant strain B. amyloliquefaciens FZB42 in the rhizosphere of lettuce. However, abundance of the strain declines in the course of the experiment.

\section{COMPARATIVE TAXONOMIC CHARACTERIZATION OF THE MICROBIAL COMMUNITIES IN THE LETTUCE RHIZOSPHERES WITH AND WITHOUT APPLICATION OF B. AMYLOLIQUEFACIENS FZB42 BY WHOLE METAGENOME SEQUENCE ANALYSES}

Determination of the $\alpha$-diversities summarizing the mean species diversity in habitats represented by the six metagenome data sets showed a clear increase from 332.82 in the control sample and 349.23 in the with FZB42 treated sample taken after at planting to 430.81 in the control sample and 447.81 in the with FZB42 treated sample taken 2 weeks after planting (Table 3 ). After 5 weeks, the $\alpha$-diversities of 412.71 in the control and 437.21 in the inoculated sample were measured.

On average, the reads of the sequenced metagenome libraries were assigned to 27 phyla, 41 classes, 92 orders, 211 families, and 596 genera within the superkingdom Bacteria (Table 4). Considering the six samples for different treatments and the three sampling time points, a total of 27 phyla, 41 classes, 95 orders, 217 families, and 689 genera were detected. Deduced taxonomic profiles clearly overlap at higher taxonomic levels and in a slightly decreasing manner at lower levels for the different samples.
These results indicate that the general compositions of the analyzed microbial communities within the lettuce rhizospheres are similar.

Analysis of the most abundant phyla within the sample sets taken at different time points revealed a high overlap resulting in only 11 phyla representing the 10 most prominent phyla within the single sample sets. The most abundant phyla Proteobacteria, Actinobacteria, Bacteroidetes, and Firmicutes represented 95.0 to 96.7\% of all assigned sequences (Table $5 \mathbf{a}$ and Figure 3 ). Within the metagenome datasets derived from the control samples and samples from inoculated rhizospheres, only minor changes are noticeable, whereas a more distinct shift between relative frequencies of assigned community taxa in the course of the experiment can be observed. The most obvious changes were detectable within the mentioned three most prominent phyla of the microbial communities after 2 weeks of cultivation in the field. The amount of annotated Proteobacteria decreased from over $74 \%$ to approximately $59 \%$ in the $\mathrm{t} 1$ samples to $64 \%$ in the $\mathrm{t} 2$ samples, and Bacteroidetes decreased from around 8\% (t0) to about 4.5\% (t1) to $6.6 \%(\mathrm{t} 2)$. The ratios of annotated Actinobacteria increased from between $9 \%$ in the control sample and $12 \%$ in the with FZB42 treated sample from t0 to around $24 \%$ within the samples taken after 2 and 5 weeks. Additionally the ratio of Firmicutes increased from around $0.4 \%$ in the to samples to around $4 \%$ within the $\mathrm{t} 1$ and then decreased again to around $2.7 \%$ in the t2 samples. The remaining frequencies of annotated phyla varied only slightly between all samples.

The 10 most abundant classes of the single data sets add up to 12 different classes represented by around $97.4 \%$ of all annotated bacterial sequences. The four most prominent classes Alphaproteobacteria, Betaproteobacteria, Actinobacteria, and Gammaproteobacteria were represented by $84.6-88.2 \%$ of all annotated sequences (Table $\mathbf{5 b}$ and Figure 4 ). Distinct changes between samples taken at different time points were detected within the first 2 weeks of cultivation, whereas the microbial rhizosphere communities were relatively stable in the last 3 weeks of cultivation. The relative abundance of annotated Betaproteobacteria that was around $30 \%$ at $\mathrm{t} 0$, decreased to $22 \%$ at $\mathrm{t} 1$ and around $20 \%$ in the $\mathrm{t} 2$ samples. The amount

Table $3 \mid \alpha$ diversities within the metagenome datasets.

\begin{tabular}{|c|c|c|c|c|c|c|}
\hline & to control & to inoculated & t1 control & t1 inoculated & t2 control & t2 inoculated \\
\hline$\alpha$ diversity & 332.82 & 349.23 & 430.81 & 447.14 & 412.71 & 437.21 \\
\hline
\end{tabular}

Table 4 | Numbers of different taxa assigned to the superkingdom Bacteria detected within the metagenome sequences as analyzed by the MG-RAST software package.

\begin{tabular}{|c|c|c|c|c|c|c|c|}
\hline & to control & to inoculated & t1 control & t1 inoculated & t2 control & t2 inoculated & sum $^{a}$ \\
\hline Phylum & 27 & 27 & 27 & 27 & 27 & 27 & 27 \\
\hline Class & 41 & 41 & 41 & 41 & 41 & 41 & 41 \\
\hline Order & 92 & 93 & 91 & 92 & 92 & 91 & 95 \\
\hline Family & 210 & 213 & 211 & 214 & 210 & 210 & 217 \\
\hline Genus & 602 & 613 & 588 & 601 & 598 & 573 & 689 \\
\hline
\end{tabular}

${ }^{a}$ Combined number of different taxa within all samples. 
Table 5 | Relative abundances of the dominant bacterial phyla (a), classes (b), orders (c), and genera (d) within the rhizosphere of $L$. sativa calculated for all classified sequences within the bacterial domain.

\begin{tabular}{|c|c|c|c|c|c|c|}
\hline & to control & to inoculated & t1 control & t1 inoculated & t2 control & t2 inoculated \\
\hline Proteobacteria & 79.35 & 74.23 & 59.31 & 60.27 & 63.74 & 64.33 \\
\hline Bacteroidetes & 7.88 & 8.92 & 4.65 & 5.26 & 5.04 & 6.62 \\
\hline Firmicutes & 0.39 & 0.56 & 4.27 & 4.07 & 2.92 & 2.54 \\
\hline Chloroflexi & 0.08 & 0.09 & 0.73 & 0.64 & 0.38 & 0.55 \\
\hline Planctomycetes & 0.18 & 0.22 & 0.53 & 0.43 & 0.36 & 0.37 \\
\hline Cyanobacteria & 0.11 & 0.13 & 0.30 & 0.25 & 0.20 & 0.25 \\
\hline Deinococcus-Thermus & 0.06 & 0.08 & 0.22 & 0.19 & 0.12 & 0.14 \\
\hline Chlorobi & 0.07 & 0.09 & 0.10 & 0.13 & 0.10 & 0.11 \\
\hline Actinobacteria & 9.07 & 12.27 & 26.99 & 26.18 & 24.10 & 22.26 \\
\hline Gammaproteobacteria & 23.99 & 17.10 & 11.63 & 8.49 & 10.70 & 10.86 \\
\hline Sphingobacteria & 4.26 & 5.15 & 2.25 & 2.28 & 3.27 & 2.96 \\
\hline Bacilli & 0.19 & 0.35 & 3.55 & 3.39 & 2.46 & 2.03 \\
\hline Flavobacteria & 2.05 & 1.91 & 1.37 & 1.92 & 0.88 & 2.65 \\
\hline Deltaproteobacteria & 0.59 & 0.70 & 2.11 & 2.12 & 1.33 & 1.52 \\
\hline Cytophagia & 1.35 & 1.61 & 0.81 & 0.86 & 0.70 & 0.78 \\
\hline Acidobacteria & 1.47 & 1.87 & 0.47 & 0.39 & 0.70 & 0.66 \\
\hline Opitutae & 0.43 & 0.55 & 0.37 & 0.35 & 0.84 & 0.65 \\
\hline Clostridia & 0.18 & 0.19 & 0.71 & 0.65 & 0.43 & 0.49 \\
\hline Sphingomonadales & 1.84 & 1.93 & 5.62 & 6.41 & 5.24 & 6.54 \\
\hline Xanthomonadales & 4.94 & 5.17 & 2.38 & 2.69 & 2.65 & 2.60 \\
\hline Sphingobacteriales & 4.30 & 5.21 & 2.27 & 2.30 & 3.32 & 3.00 \\
\hline Bacillales & 0.16 & 0.32 & 3.51 & 3.34 & 2.45 & 1.99 \\
\hline Flavobacteriales & 2.03 & 1.90 & 1.36 & 1.91 & 0.87 & 2.65 \\
\hline Enterobacteriales & 3.05 & 1.94 & 2.00 & 1.14 & 0.75 & 0.90 \\
\hline Methylophilales & 1.11 & 1.07 & 0.65 & 0.56 & 2.08 & 2.22 \\
\hline Others & 9.89 & 11.44 & 13.50 & 13.11 & 11.16 & 11.95 \\
\hline \multicolumn{7}{|l|}{ (d) GENUS } \\
\hline Mycobacterium & 3.89 & 7.42 & 3.82 & 3.80 & 5.67 & 4.42 \\
\hline Pseudomonas & 5.55 & 5.04 & 3.70 & 2.20 & 5.53 & 5.39 \\
\hline Burkholderia & 8.07 & 6.86 & 2.71 & 2.66 & 3.13 & 2.73 \\
\hline Caulobacter & 5.10 & 5.18 & 2.64 & 2.37 & 4.17 & 4.43 \\
\hline Acidovorax & 3.28 & 3.51 & 2.98 & 4.09 & 2.32 & 2.81 \\
\hline Variovorax & 1.77 & 1.82 & 3.65 & 4.56 & 3.19 & 3.00 \\
\hline Streptomyces & 0.39 & 0.47 & 3.36 & 3.37 & 4.47 & 3.80 \\
\hline Arthrobacter & 0.69 & 0.56 & 5.45 & 5.44 & 1.66 & 1.58 \\
\hline Rhodopseudomonas & 1.85 & 2.42 & 2.22 & 2.34 & 3.23 & 3.19 \\
\hline
\end{tabular}


Table 5 | Continued

\begin{tabular}{|c|c|c|c|c|c|c|}
\hline & to control & to inoculated & t1 control & t1 inoculated & t2 control & t2 inoculated \\
\hline Polaromonas & 2.81 & 3.25 & 1.98 & 2.45 & 2.14 & 2.12 \\
\hline Sphingomonas & 0.61 & 0.64 & 2.44 & 2.78 & 2.24 & 2.99 \\
\hline Xanthomonas & 2.45 & 2.59 & 1.18 & 1.37 & 1.32 & 1.37 \\
\hline Others & 51.82 & 54.02 & 55.49 & 55.60 & 54.76 & 56.12 \\
\hline
\end{tabular}

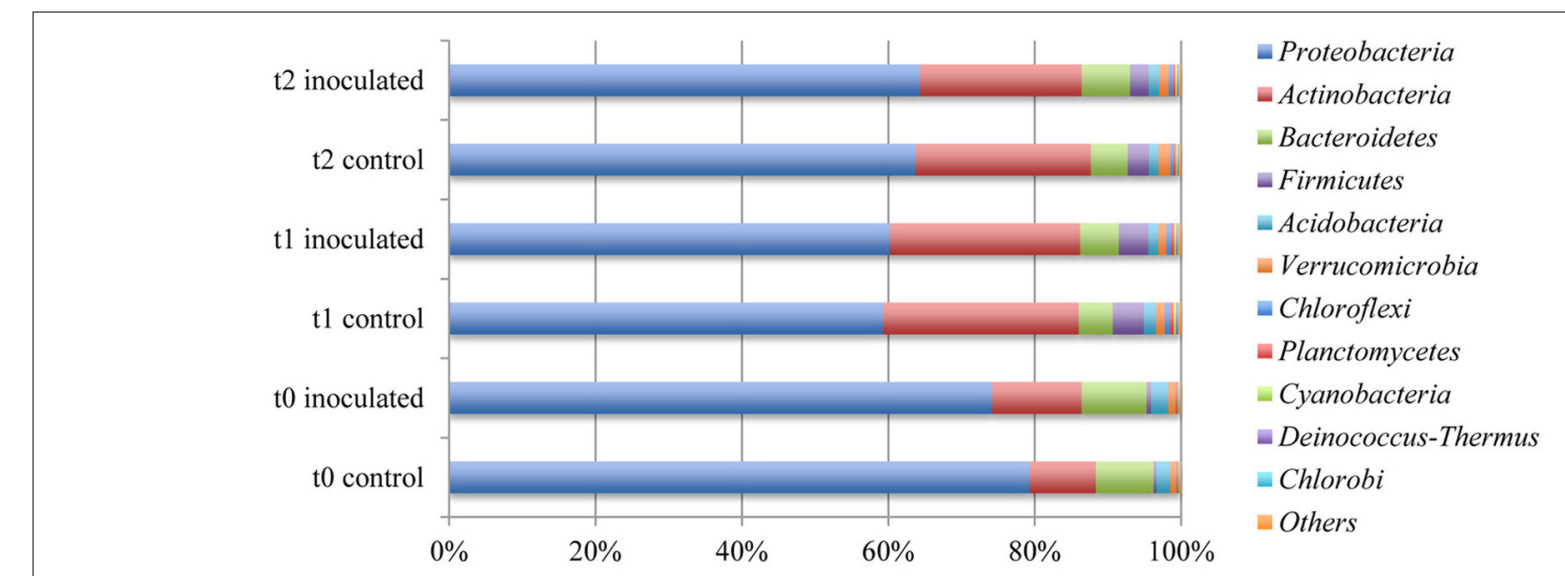

FIGURE 3 | Comparison of the ten most abundant phyla within the rhizosphere of lettuce. Abundances were calculated by means of comparison of metagenome sequence reads against the GenBank database within the MG-RAST software package.

of annotated Actinobacteria increased noticeably from between 9 and $12 \%$ to around $24.8 \%$ in average in the samples drawn after 2 and 5 weeks. The relative abundances of annotated Gammaproteobacteria varied between the t0 samples between $24 \%$ in the control and $17 \%$ in the inoculated samples. Within the samples taken after 2 and 5 weeks of cultivation in the field the ratio of Gammaproteobacteria was around 10\%.

Analysis of the most abundant orders revealed that Burkholderiales, Actinomycetales, Rhizobiales, and Pseudomonadales represented between $62 \%$ and almost $66 \%$ of the microbial communities in all six samples that where either inoculated or not treated (Table 5c and Figure 5). A decrease from around $28.5 \%$ annotated Burkholderiales in the t0 sample to an average of $19.3 \%$ in the other samples was noticeable. The ratios of annotated Actinomycetales increased from around 10\% in the first samples to over $20 \%$ after 2 and 5 weeks of cultivation. The differences between samples collected at different time points were more distinct as compared to samples for different treatments.

The most obvious change probably caused by the application of strain FZB42 was the relative abundance of annotated Pseudomonadales varying between 14.5 and $8.3 \%$ in the samples taken at planting. The amount decreases to $6.0 \%$ in the control and $3.3 \%$ in the with FZB42 treated sample after 2 weeks and around $6 \%$ in both samples drawn after 5 weeks.
The 10 most prominent genera within the samples taken from different time points add up to 16 genera representing around $45 \%$ of all annotated sequences. Mycobacterium, Pseudomonas, Burkholderia, and Caulobacter represented the most abundant genera within the microbial rhizosphere communities (Table 5d and Figure 6). Among the ratios for Mycobacteria, an increase from 3.9 to $7.4 \%$ was detectable for the control and the with FZB42 treated samples of $\mathrm{t} 0$, whereas the ratios were almost stable among the remaining samples that were either treated with FZB42 or not treated. Relative abundances for other genera revealed more pronounced changes between the samples that were taken at different time points than between the differentially treated samples. M/A plots of the annotated metagenome sequences on genus level visualized the distributions of detected genera clearly (Figures 7-9). The dense clouds around zero on the $y$-axis of these plots indicated a low fold-change in the abundances of annotated genera between the control and the inoculated samples. Higher variations were only noticeable within the genera that were annotated at very low abundances and were therefore of minor interest, because they do not contribute to the main share of the microbial communities. Within the t0 control sample a higher number of sequences were classified as Acinetobacter, Enterobacter, and Citrobacter in comparison to the inoculated sample. In the inoculated sample from this time point, the number of annotated Bacillus, Pantoea, and 


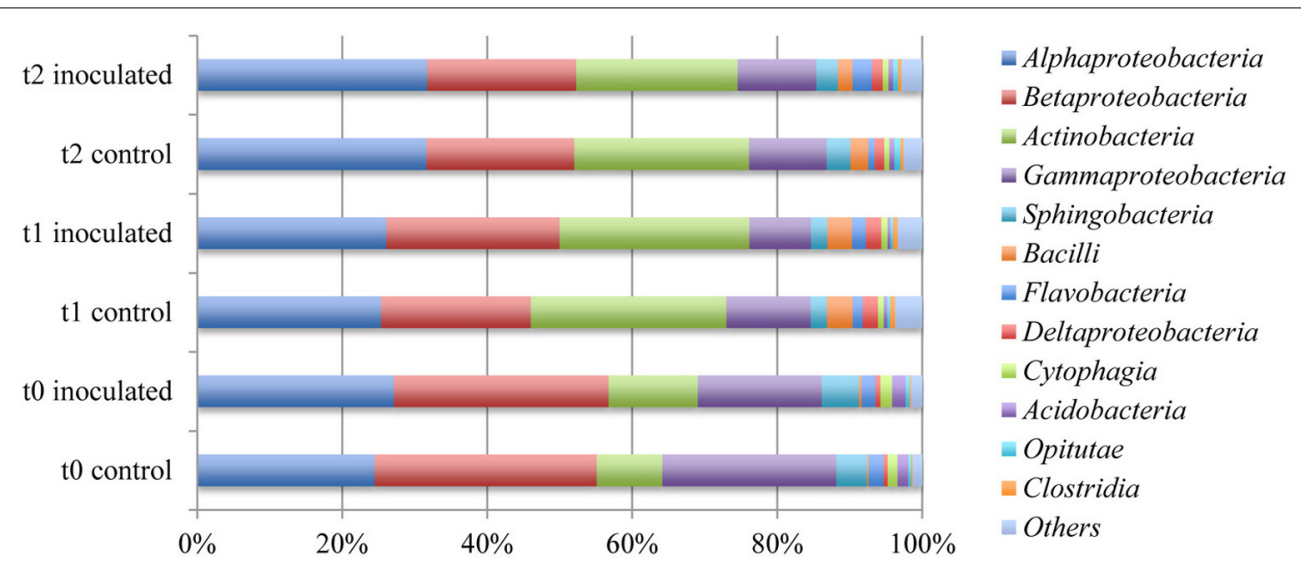

FIGURE 4 | Comparison of the ten most abundant classes within the rhizosphere of lettuce. Abundances were calculated by means of comparison of metagenome sequence reads against the GenBank database within the MG-RAST software package.

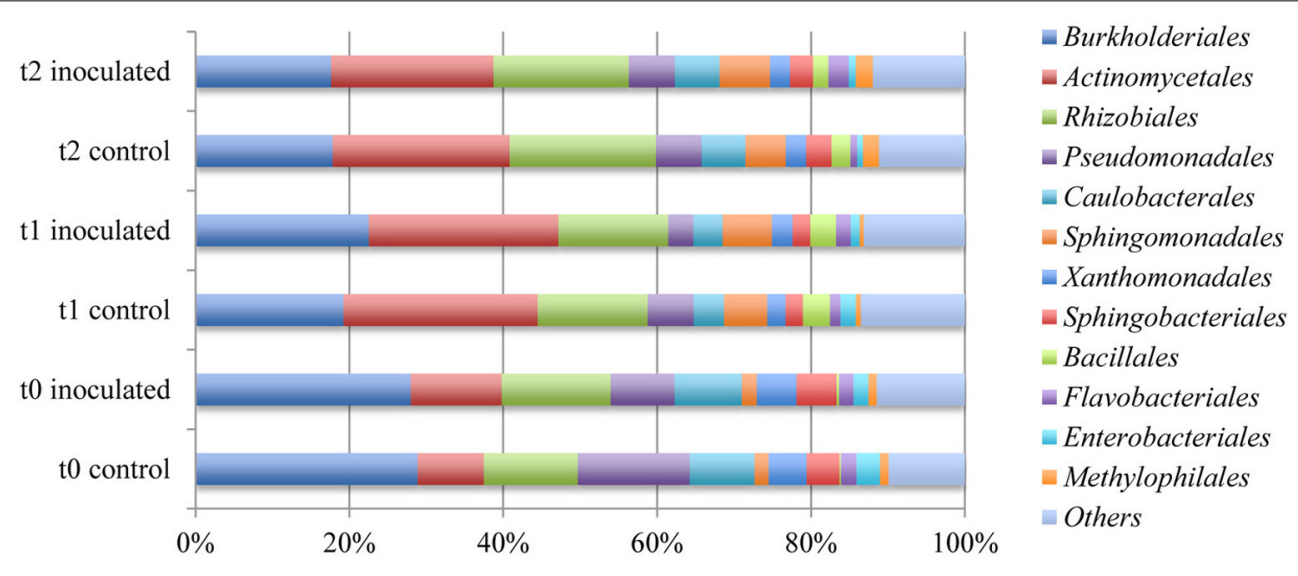

FIGURE 5 | Comparison of the ten most abundant orders within the rhizosphere of lettuce. Abundances were calculated by means of comparison of metagenome sequence reads against the GenBank database within the MG-RAST software package.

Mycobacterium was increased (Figure 7). The samples taken after 2 weeks of cultivation in the field showed even less noticeable differences (Figure 8). Only the abundances of Acinetobacter and Enterobacter were slightly increased in the control samples. Within the samples of $\mathrm{t} 2$ only the genus Flavobacterium was detected in a higher abundance in the inoculated sample (Figure 9).

Accordingly, application of $B$. amyloliquefaciens FZB42 appeared to have almost no effect on composition of the lettuce rhizosphere communities, whereas the growth states of the analyzed plants seem to alter the composition of the communities in a more noticeable manner.

\section{ANALYSIS OF THE POTENTIAL IMPACT OF B. AMYLOLIQUEFACIENS FZB42 ON ANNOTATED FUNCTIONAL SUBSYSTEMS OF MICROBIAL COMMUNITY MEMBERS IN THE LETTUCE RHIZOSPHERES}

In order to gain further insights into community changes at the functional level, classifications of annotated genes according to functional subsystems as defined within MG-RAST of all samples were compared. The ratios of sequences assigned to any subsystem decreased from around $74.6 \%$ in the t0 samples to around $62 \%$ within the control samples and $57 \%$ within the inoculated samples taken after 2 and 5 weeks of cultivation in the field (Table 6). The main focus was put on the carbohydrate, nitrogen and amino acid metabolic pathways, since these subsystems are important for utilization and metabolism of plant root exudates.

Examination of the relative amounts of metagenome sequences assigned to specific subsystems for different samples did not show any pronounced changes neither between with FZB42 treated or non-treated samples nor over the duration of the cultivation (see Table 7). Closer examination of the annotated genes within these mentioned functional subsystems of the samples also revealed very similar sets of assigned genes.

Within the functional subclass of carbohydrate metabolism, some minor changes were detected between the samples taken at different time points (Table 8). Frequencies of detected genes associated with the metabolism of di-, oligo-, and polysaccharides increased slightly in the samples taken after 2 and 5 weeks of cultivation. Additionally, the frequencies of annotated genes involved in the metabolism of different organic acids decrease slightly from 


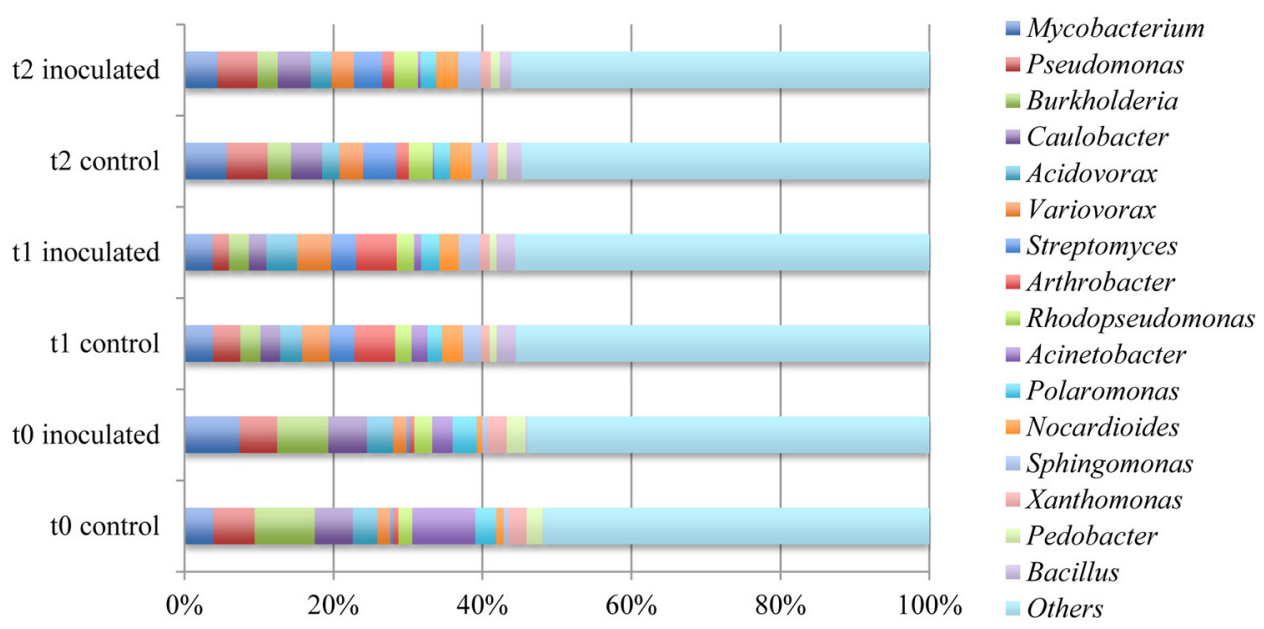

FIGURE 6 | Comparison of the ten most abundant genera within the rhizosphere of lettuce. Abundances were calculated by means of comparison of metagenome sequence reads against the GenBank database within the MG-RAST software package.

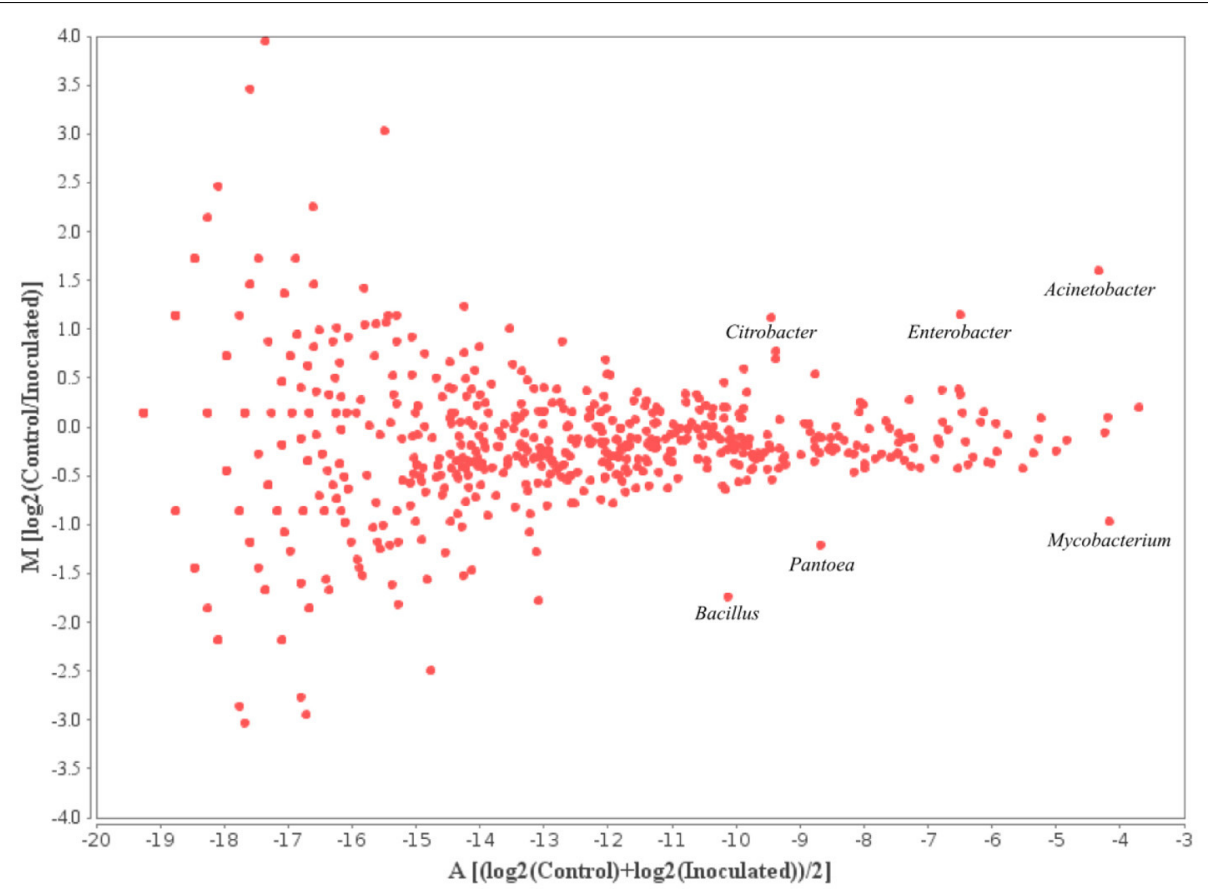

FIGURE 7 | MA-Plot visualizing the values of annotated genera within the to samples. Log2(Control/Inoculated) indicates the fold-changes in the abundances of annotated genera between the two samples; (log2(Control)+log2(Inoculated))/2 shows the average abundance of an annotated genus.

around $6.1 \%$ within the samples taken after 2 days to $5.5 \%$ for the samples taken after 2 and 5 weeks.

Frequencies of several annotated genes within the functional subclass of nitrogen metabolism are probably influenced by either the growth state of the plants or the transfer of plants into the field (Table 9). Genes involved in ammonia assimilation are more prominent within the first sample. Their frequencies decrease from around $47 \%$ at planting to values between 44 and $45 \%$ after 2 and 5 weeks. The ratios of genes within the annotated subsystem of nitrosative stress decreased in the course of the cultivation from $5.7 \%$ to around $5 \%$ and $4.4 \%$. In contrast, frequencies of genes within the subsystem of nitrate and nitrite ammonification increased slightly during the course of the cultivation. Additionally, genes involved in nitrogen fixation increased in a similar manner.

Within the functional subsystem of amino acid and amino acid derivative metabolism, the calculated frequencies of annotated subsystems almost showed no changes (Table 10). The most 


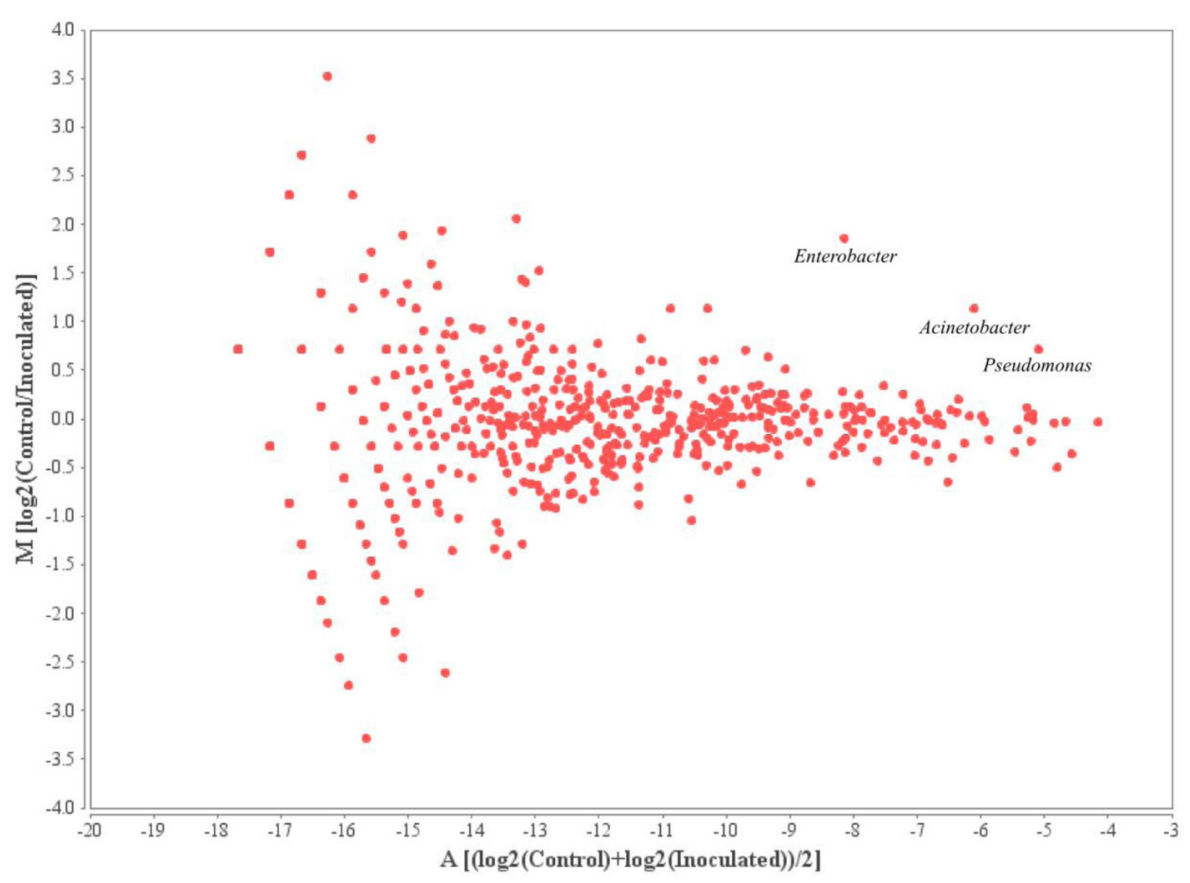

FIGURE 8 | MA-Plot visualizing the values of annotated genera within the $\mathbf{t} 1$ samples. Log2(Control/Inoculated) indicates the fold-changes in the abundances of annotated genera between the two samples; (log2(Control) $+\log 2$ (Inoculated))/2 shows the average abundance of an annotated genus.

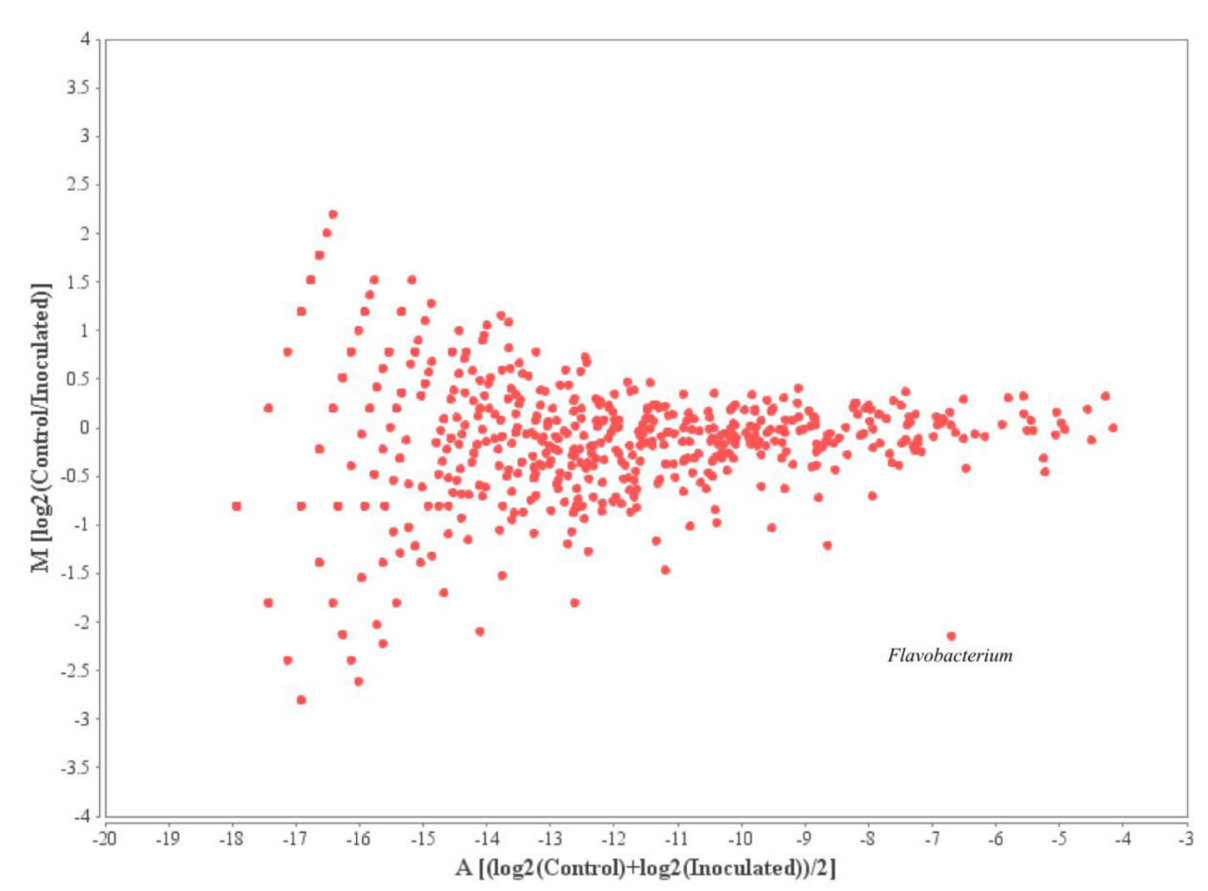

FIGURE 9 | MA-Plot visualizing the values of annotated genera within the $\mathbf{t} 2$ samples. Log2(Control/lnoculated) indicates the fold-changes in the abundances of annotated genera between the two samples; (log2(Control) $+\log 2($ Inoculated) $) / 2$ shows the average abundance of an annotated genus.

noticeable changes occurred in the subsystem of glutamine, glutamate, aspartate, asparagine and ammonia assimilation with frequencies increasing from around $10.3 \%$ to values around $11 \%$ in the samples taken after 2 and 5 weeks.
Additionally, metagenome sequences assigned to the functional context "defense mechanisms" such as antibiotic resistances and transport systems for toxic compounds that may be indicative for microorganisms resistant to secondary metabolites produced 
Table 6 | Sequences assigned to all functional subclasses within the metagenome data sets calculated within the MG RAST analysis software with default settings.

\begin{tabular}{|c|c|c|c|c|c|c|}
\hline & to control & to inoculated & t1 control & t1 inoculated & t2 control & t1 inoculated \\
\hline HO sequences & $1,869,056$ & $2,446,824$ & 783,195 & $1,470,887$ & $1,550,845$ & 971,074 \\
\hline \multirow[t]{2}{*}{ Assigned sequences } & $1,419,743$ & $1,791,952$ & 485,868 & 837,363 & 967,685 & 557,903 \\
\hline & $75.96 \%$ & $73.24 \%$ & $62.04 \%$ & $56.93 \%$ & $62.40 \%$ & $57.45 \%$ \\
\hline
\end{tabular}

Table 7 | Relative distributions of functional subsystems calculated within the MG RAST analysis software with default settings.

\begin{tabular}{|c|c|c|c|c|c|c|}
\hline Functional subsystems & to control & to inoculated & t1 control & t1 inoculated & t2 control & t2 inoculated \\
\hline Carbohydrates & 10.2 & 10.1 & 10.8 & 11.0 & 10.7 & 10.9 \\
\hline Cell wall and capsule & 3.8 & 3.7 & 3.5 & 3.4 & 3.5 & 3.5 \\
\hline Clustering-based subsystems & 14.7 & 14.8 & 15.2 & 15.1 & 15.1 & 15.0 \\
\hline Cofactors, vitamins, prosthetic groups, pigments & 6.6 & 6.7 & 6.8 & 6.8 & 6.9 & 6.9 \\
\hline Dormancy and sporulation & 0.2 & 0.2 & 0.2 & 0.2 & 0.2 & 0.2 \\
\hline Fatty acids, lipids, and isoprenoids & 3.1 & 3.2 & 3.2 & 3.1 & 3.2 & 3.2 \\
\hline Iron acquisition and metabolism & 1.2 & 1.1 & 0.7 & 0.7 & 0.8 & 0.8 \\
\hline Membrane transport & 3.5 & 3.4 & 3.2 & 3.1 & 3.2 & 3.2 \\
\hline Metabolism of aromatic compounds & 2.1 & 2.0 & 2.0 & 2.0 & 2.0 & 2.1 \\
\hline Miscellaneous & 8.4 & 8.4 & 8.4 & 8.3 & 8.3 & 8.3 \\
\hline Phages, prophages, plasmids, transposable elements & 1.5 & 1.6 & 1.5 & 1.5 & 1.4 & 1.4 \\
\hline Phosphorus metabolism & 0.9 & 0.9 & 0.9 & 0.9 & 0.8 & 0.8 \\
\hline Photosynthesis & 0.1 & 0.1 & 0.1 & 0.1 & 0.1 & 0.1 \\
\hline Potassium metabolism & 0.5 & 0.5 & 0.4 & 0.4 & 0.5 & 0.4 \\
\hline Protein metabolism & 7.6 & 7.7 & 7.5 & 7.7 & 7.6 & 7.5 \\
\hline RNA metabolism & 4.1 & 4.1 & 3.9 & 3.9 & 3.9 & 3.9 \\
\hline Regulation and cell signaling & 1.5 & 1.5 & 1.5 & 1.5 & 1.5 & 1.5 \\
\hline Respiration & 3.4 & 3.4 & 3.6 & 3.5 & 3.5 & 3.6 \\
\hline Secondary metabolism & 0.4 & 0.4 & 0.4 & 0.5 & 0.5 & 0.4 \\
\hline Stress response & 2.7 & 2.7 & 2.6 & 2.7 & 2.7 & 2.7 \\
\hline Sulfur metabolism & 1.3 & 1.3 & 1.3 & 1.3 & 1.3 & 1.3 \\
\hline
\end{tabular}

Table 8 | Relative abundances of annotated subsystems within the annotated functional system of carbohydrate metabolism.

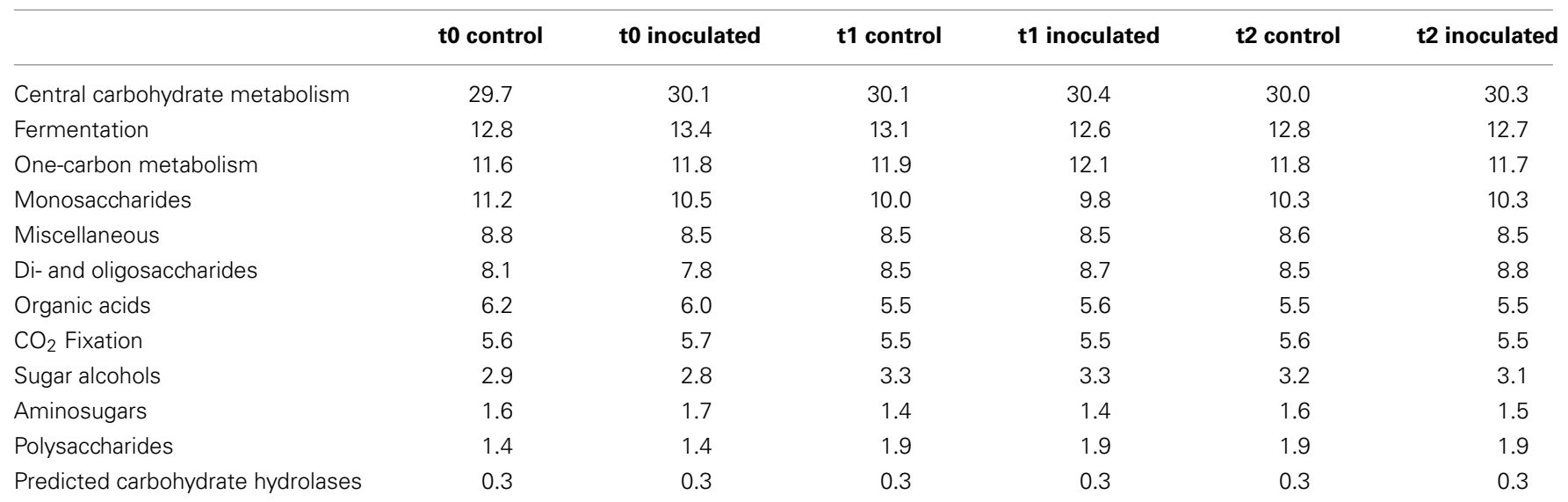


by $B$. amyloliquefaciens FZB42 were analyzed (Table 11). The relative abundances of sequences corresponding to this subsystem varied between 2.8 and $3 \%$ of all assigned sequences within each sample. Examination of the subgroups of the subsystem "defense mechanisms" substantiates the conclusion that application of B. amyloliquefaciens FZB42 does not affect rhizosphere microorganisms featuring functions in the context of defense.

In summary, comparison of the relative abundances of sequences assigned to sub-systems between samples neither showed pronounced differences between the inoculated and nontreated samples nor between the samples taken over the course of the field trial. Therefore, no major impact of the inoculant strain FZB42 on functional community profiles could be affirmed.

\section{DISCUSSION}

\section{B. AMYLOLIQUEFACIENS FZB42 IS ABLE TO SUCCESSFULLY COLONIZE THE LETTUCE RHIZOSPHERE IN THE COURSE OF THE FIELD EXPERIMENT}

Tracking of the inoculant strain B. amyloliquefaciens FZB42 in the lettuce rhizosphere in the course of the experiment was of crucial importance to estimate its competitiveness in this habitat and was achieved by mapping of metagenome sequences to the published reference genome of $B$. amyloliquefaciens FZB42. Corresponding fragment recruitments proved persistence of strain FZB42 in the rhizosphere. However, the number of mapped reads (contigs) and the number of covered B. amyloliquefaciens FZB42 genes decreased in the course of the plant cultivation indicating that the

Table 9 | Relative abundances of annotated subsystems within the annotated functional system of nitrogen metabolism.

\begin{tabular}{|c|c|c|c|c|c|c|}
\hline & to control & to inoculated & t1 control & t1 inoculated & t 2 control & t2 inoculated \\
\hline Ammonia assimilation & 47.2 & 47.1 & 44.1 & 43.3 & 44.7 & 45.3 \\
\hline Nitrate and nitrite ammonification & 21.0 & 19.8 & 22.1 & 21.4 & 22.3 & 22.4 \\
\hline Denitrification & 8.5 & 8.4 & 7.4 & 8.2 & 6.9 & 7.1 \\
\hline Allatoin utilization & 5.7 & 5.5 & 7.1 & 6.1 & 5.4 & 5.2 \\
\hline Nitrosative stress & 5.7 & 5.7 & 5.1 & 5.0 & 4.6 & 4.2 \\
\hline Nitric oxide synthase & 4.7 & 5.8 & 6.5 & 6.5 & 7.6 & 7.3 \\
\hline Dissimilatory nitrite reductase & 3.2 & 3.3 & 3.0 & 3.7 & 3.5 & 3.5 \\
\hline Cyanite hydrolysis & 3.2 & 3.4 & 3.1 & 3.5 & 3.6 & 3.2 \\
\hline Nitrogen fixation & 0.4 & 0.7 & 1.2 & 1.7 & 1.2 & 1.4 \\
\hline Amidase cluster & 0.3 & 0.3 & 0.2 & 0.3 & 0.3 & 0.4 \\
\hline Nitrilase & 0.0 & 0.1 & 0.1 & 0.1 & 0.0 & 0.1 \\
\hline
\end{tabular}

Table 10 | Relative abundances of annotated subsystems within the annotated functional system of amino acids and derivatives.

\begin{tabular}{|c|c|c|c|c|c|c|}
\hline & to control & to inoculated & t1 control & t1 inoculated & t2 control & t2 inoculated \\
\hline Branched-chain amino acids & 28.7 & 29.9 & 28.3 & 29.1 & 28.8 & 29.5 \\
\hline Lysine, threonine, methionine, and cysteine & 21.4 & 21.7 & 21.2 & 21.4 & 21.3 & 21.4 \\
\hline Aromatic amino acids and derivatives & 11.5 & 11.2 & 10.9 & 10.5 & 10.7 & 10.9 \\
\hline Arginine; urea cycle, polyamines & 11.0 & 10.5 & 11.2 & 11.0 & 11.1 & 11.0 \\
\hline $\begin{array}{l}\text { Glutamine, glutamate, aspartate, asparagine; ammonia } \\
\text { assimilation }\end{array}$ & 10.4 & 10.2 & 11.4 & 11.2 & 11.1 & 10.7 \\
\hline Alanine, serine, and glycine & 9.8 & 9.6 & 10.2 & 10.0 & 10.1 & 10.0 \\
\hline Proline and 4-hydroxyproline & 3.7 & 3.5 & 3.1 & 3.0 & 3.1 & 3.0 \\
\hline Histidine metabolism & 2.9 & 2.7 & 2.8 & 2.7 & 2.7 & 2.7 \\
\hline Miscellaneous & 0.7 & 0.7 & 1.1 & 1.1 & 1.1 & 1.0 \\
\hline
\end{tabular}

Table 11 | Relative abundances of annotated subsystems within the annotated functional system of Virulence, disease, and defense.

\begin{tabular}{|c|c|c|c|c|c|c|}
\hline & to control & to inoculated & t1 control & t1 inoculated & t2 control & t2 inoculated \\
\hline Resistance to antibiotics and toxic compounds & 80.7 & 81.4 & 78.9 & 78.8 & 79.7 & 79.8 \\
\hline- & 9.7 & 9.1 & 10.3 & 10.2 & 10.4 & 10.2 \\
\hline Multi locus sequence typing & 7.2 & 7.2 & 8.2 & 8.4 & 7.5 & 7.4 \\
\hline Adhesion & 1.4 & 1.4 & 1.5 & 1.5 & 1.5 & 1.5 \\
\hline Bacteriocins, ribosomally synthesized antibacterial peptides & 0.4 & 0.5 & 0.6 & 0.5 & 0.4 & 0.6 \\
\hline Invasion and intracellular resistance & 0.4 & 0.3 & 0.3 & 0.3 & 0.3 & 0.3 \\
\hline Toxins and superantigens & 0.1 & 0.1 & 0.2 & 0.2 & 0.2 & 0.1 \\
\hline
\end{tabular}


inoculant strain was less competitive compared to other community members. At the end of the experiment, strain FZB42 could still be traced at a clear level. Corrected and normalized values of the read numbers matching specific B. amyloliquefaciens FZB42 genes revealed that after 2 weeks $64 \%$ and after 5 weeks about 55\% of the inoculant FZB42 was still traceable within the rhizosphere. These values are in accordance with previous results from reference experiments using a FZB42-derivative conferring rifampicin (Rif) resistance as inoculum. The CFUs per gram of soil of this strain decreased to $59 \%$ after 2 weeks and to $14 \%$ after 5 weeks of field cultivation (Chowdhury et al., 2013). The relatively high rate of recruited metagenome sequences from the non-inoculated samples is most probably due to the fact that sequences of rRNA genes and mobile genetic elements are very similar or even identical between different Bacillus species (Edwards et al., 2012). Accordingly, increasing numbers of mapped reads from the noninoculated samples can be explained by the presence of other indigenous Bacillus species in the plant rhizosphere.

\section{INOCULATION WITH B. AMYLOLIQUEFACIENS FZB42 HAS NO MAJOR IMPACT ON THE MICROBIAL COMMUNITIES IN THE LETTUCE RHIZOSPHERE AND ON THE FUNCTIONAL SUBSYSTEM ASSIGNMENTS OF COMMUNITY MEMBERS}

Although competitiveness of B. amyloliquefaciens FZB42 in the lettuce rhizosphere is limited, application of the strain may affect composition of the indigenous microbial rhizosphere community, especially in early stages of plant development. Metagenomic sequencing and the subsequent comparison of deduced taxonomic profiles combining samples from several equally treated plant rhizospheres indicated that application of B. amyloliquefaciens FZB42 did not result in pronounced effects on the composition of the microbial communities of the lettuce rhizosphere. Microbial community changes seem more distinct between the samples drawn at different time points. Most obvious, changes in the composition of the microbial communities occur within the first 2 weeks of plant cultivation. It is known that plant root exudates have a major impact on the composition of microbial rhizosphere communities (Hartmann et al., 2009). Root exudation patterns change quantitatively and qualitatively during the growth of the plant and higher amounts of exudates were released into the rhizosphere usually at the initial growth phase as given after planting (Baudoin et al., 2002). Hence, changes in root exudate patterns may be responsible for a shift in microbial community structure in the course of plant growth. Similar studies showed that shifts within microbial rhizosphere communities are mainly due to different developmental stages of the plant (Inceoglu et al., 2013) and root exudates produced by the plants (Chaparro et al., 2013). In the study described here, community changes were most pronounced at the onset of cultivation whereas comparison of the taxonomic profiles between the samples after 2 and 5 weeks of plant growth revealed only minor differences in the ratios of identified taxa suggesting that environmental conditions in this time period are almost constant and therefore enable the establishment of an adapted bacterial community in the rhizosphere.

Comparison of the taxonomic profiles within the lettuce rhizosphere with other soil microbial communities shows similar compositions. Several studies on related habitats revealed the same five most abundant phyla Acidobacteria, Bacteroidetes, Actinobacteria, Firmicutes, and Proteobacteria with Firmicutes and Proteobacteria being the most prominent taxa in these microbial communities. Referenced studies include the analysis of the rhizosphere of Lotus japonicus (Unno and Shinano, 2013), the rhizosphere of sugarcane plants (Pisa et al., 2011) and the analysis of several soil metagenome samples via 16S rRNA gene amplicon sequencing (Vasileiadis et al., 2012).

Evaluation of assignments to functional subsystems of community members supported the results of the taxonomic classification. Similar to the taxonomic profiles, a shift over the course of the cultivation but not between the control and the with FZB42 treated samples was observed. Most obvious was the decrease within the ratio of sequences that could generally be assigned to any subsystem over the course of the study.

Changes within the functional subgroups that are associated with the metabolism of different saccharides, organic acids or amino acids are probably due to changes within the composition of root exudates from the lettuce plants (Baudoin et al., 2002). An additional factor for the slight decrease in the relative number of genes associated with the metabolism of organic acids after 2 and 5 weeks of cultivation probably was the transfer of plants into the field. The plants were grown in peat blocks that probably contained a higher amount of organic acids such as humic acids.

The detected changes corresponding to genes associated with nitrogen metabolism can probably be traced back to reactions of the lettuce plants to several abiotic stress factors (Molassiotis and Fotopoulos, 2011) such as reactive nitrogen species (RNS) including $\mathrm{NO}$ and other derivatives. The increase in the number of annotated nitrogen fixation genes may be ascribed to limited amounts of nitrogen in the soil and therefore the need to utilize atmospheric dinitrogen as nitrogen source. Additionally, nitrogen fixation has been proven to be a factor in the promotion of plant growth (Bulgarelli et al., 2013).

Although metagenome analysis is a very convenient method to determine the composition and functional profile of microbial rhizosphere communities only very few published studies applied this approach. Most of the earlier studies are based on T-RFLP and 16S rRNA gene amplicon sequencing which complicates direct comparison of the results obtained in this study. In addition to the inadequacy of comparing results obtained by applying different techniques, most studies only provide taxonomic profiles down to the level of orders. Therefore comparisons of microbial communities within different rhizospheres down to lower taxonomic levels are a relatively challenging task and demonstrate the need to perform further metagenome studies to get deeper insights in microbial communities associated with plants.

\section{CONCLUDING REMARKS}

Metagenome analyses of lettuce rhizosphere samples inoculated with B. amyloliquefaciens FZB42 vs. non-treated samples revealed that the inoculant strain only had a minor impact on the community structure within this habitat and on specific functional subsystems of community members. The fact that the number 
of sequences specifically matching the B. amyloliquefaciens FZB42 genome decreased during the growth period of lettuce indicated that the inoculant strain did not overgrow other relevant species within the lettuce rhizosphere.

In previously published studies, the described set of secondary metabolites produced by strain FZB42 was proven to mediate antibacterial and antifungal properties (Koumoutsi et al., 2004; Chen et al., 2006). These metabolites were suspected to induce changes within the microbial rhizosphere community, noticeable i.a., by shifts within the annotated functional subsystem of defense and resistance mechanisms. However, sequence analysis revealed only marginal changes within this subsystem so that it is likely that secondary metabolites do not have a major impact on phytopathogens in the rhizosphere and thus probably are not the key factor in the protection of the plant from pathogenic microorganisms. It should also be mentioned here that B. amyloliquefaciens FZB42 probably had not reached a critical density in the lettuce rhizosphere to exert its antibacterial and antifungal properties. Hence, other properties of the inoculant strain such as affecting the plant's ISR may have a more important impact. Studies addressing corresponding mechanisms should be performed to elucidate the mode of action of plant-growthpromoting rhizobacteria on the plant leading to prevention of infections by pathogens.

The results of this metagenome study suggest that the application of the commercially available inoculant strain FZB42 can be considered as a safe method to promote the health of the economically important lettuce plant and reduce severity of infections by phytopathogens like R. solani.

\section{AUTHOR CONTRIBUTIONS}

The authors Magdalena Kröber, Daniel Wibberg, Rita Grosch, Soumitra P. Chowdhury, Felix Eikmeyer, and Bart Verwaaijen contributed to this work with either relevant experimental work, data analysis or writing, and revising the article critically for important intellectual content. The work was supervised by Andreas Schlüter, Anton Hartmann, and Alfred Pühler. All authors approved the final version to be published and agreed to be accountable for all aspects of the work in ensuring that questions related to the accuracy or integrity of any part of the work are appropriately investigated and resolved.

\section{ACKNOWLEDGMENTS}

This work was part of the PathControl cooperative project 'Biological control of Bacillus amyloliquefaciens on the soilborne fungal pathogen $R$. solani (FKZ 0315654B) funded by the BMBF. Additionally, the bioinformatics and technological support of the Bioinformatics and Genomics Platforms at the Center for Biotechnology (CeBiTec, Bielefeld University) is gratefully acknowledged. Andreas Schlüter acknowledges the METAEXPLORE grant of the European Commission (KBBE222625).

\section{SUPPLEMENTARY MATERIAL}

The Supplementary Material for this article can be found online at: http://www.frontiersin.org/journal/10.3389/fmicb.2014. 00252/abstract

\section{REFERENCES}

Alabouvette, C., Olivain, C., and Steinberg, C. (2006). Biological control of plant diseases: the European situation. Eur. J. Plant Pathol. 114, 329-341. doi: 10.1007/s10658-005-0233-0

Andrews, M., Cripps, M. G., and Edwards, G. R. (2012). The potential of beneficial microrganims in agricultural systems. Ann. Appl. Biol. 160, 1-5. doi: 10.1111/j.1744-7348.2011.00519.x

Arguelles-Arias, A., Ongena, M., Halimi, B., Lara, Y., Brans, A., Joris, B., et al. (2009). Bacillus amyloliquefaciens GA1 as a source of potent antibiotics and other secondary metabolites for biocontrol of plant pathogens. Microb. Cell Fact. 8:63. doi: 10.1186/1475-2859-8-63

Asaka, O., and Shoda, M. (1996). Biocontrol of Rhizoctonia solani damping-off of tomato with bacillus subtilis RB14. Appl. Environ. Microbiol. 62, 4081-4085.

Aziz, R. K., Bartels, D., Best, A., DeJongh, M., Disz, T., Edwards, R., et al. (2008). The RAST Server: rapid annotations using subsystems technology. BMC Genomics 9:75. doi: 10.1186/1471-2164-9-75

Barret, M., Morrissey, J. P., and O’Gara, F. (2011). Functional genomics analysis of plant growth-promoting rhizobacterial traits involved in rhizosphere competence. Biol. Fertil. Soils 47, 729-743. doi: 10.1007/s00374-011-0605-x

Baudoin, E., Benizri, E., and Guckert, A. (2002). Impact of growth stage on the bacterial community strzcture along maize roots, as determined by metabolic and genetic fingerprinting. Appl. Soil Ecol. 19, 135-145. doi: 10.1016/S09291393(01)00185-8

Benson, D. A., Karsch-Mizrachi, I., Lipman, D. J., Ostell, J., Rapp, B. A., and Wheeler, D. L. (2011). GenBank. Nucl. Acids Res. 3, D32-D37. doi: 10.1093/nar/gkq1079

Bulgarelli, D., Schlaeppi, K., Spaepen, S., Ver Loren van Themaat, E., and SchulzeLefert, P. (2013). Structure and functions of the bacterial microbiota of plants. Annu. Rev. Plant Biol. 64, 807-838. doi: 10.1146/annurev-arplant-050312120106

Chaparro, J. M., Badri, D., Bakker, M., Suglyama, A., Manter, D., and Vivanco, J. (2013). Root exudation of phytochemicals in Arabidopsis follows specific patterns that are developmentally programmed and correlate with soil microbial functions. PLoS ONE 8:e55731. doi: 10.1371/journal.pone.0055731

Chellemi, D. O. (2002). Nonchemical management of soilborne pests in fresh market vegetable production systems. Phytopathology 92, 1367-1372. doi: 10.1094/PHYTO.2002.92.12.1367

Chen, X. H., Vater, J., Piel, J., Franke, P., Scholz, R., Schneider, K., et al. (2006). Structural and functional characterization of three polyketide synthase gene clusters in Bacillus amyloliquefaciens FZB 42. J. Bacteriol. 188, 4024-4036. doi: 10.1128/JB.00052-06

Chen, X. H., Koumoutsi, A., Scholz, R., Eisenreich, A., Schneider, K., Heinemeier, I., et al. (2007). Comparative analysis of the complete genome sequence of the plant growth-promoting bacterium Bacillus amyloliquefaciens FZB42. Nat. Biotechnol. 25, 1007-1014. doi: 10.1038/nbt1325

Choudhary, D. K., and Johri, B. N. (2009). Interactions of Bacillus spp. and plantswith special reference to induced systemic resistance (ISR). Microbiol. Res. 164, 493-513. doi: 10.1016/j.micres.2008.08.007

Chowdhury, S. P., Dietel, K., Rändler, M., Schmid, M., Junge, H., Boriss, R., et al. (2013). Effects of Bacillus amyloliquefaciens FZB42 on lettuce growth and health under pathogen pressure and its impact on the rhizosphere bacterial community. PLoS ONE 8:e68818. doi: 10.1371/journal.pone.0068818

Edwards, K. J., Logan, J. M. J., Langham, S., Swift, C., and Gharbia, S. E. (2012). Utility of real-time amplification of selected 16S rRNA gene sequences as a tool for detection and identification of microbial signatures directly from clinical samples. J. Med. Microbiol. 61, 645-652. doi: 10.1099/jmm.0. 041764-0

Ghirardi, S., Dessaint, F., Mazurier, S., Corberand, T., Raaijmakers, J. M., Meyer, J.M., et al. (2012). Identification of traits shared by rhizosphere-competent strains of fluorescent pseudomonads. Microb. Ecol. 64, 725-737. doi: 10.1007/s00248012-0065-3

Grosch, R., Junge, H., Krebs, B., and Bochow, H. (1999). Use of Bacillus subtilis as a biocontrol agent. III. Influence of Bacillus subtilis on fungal root diseases and on yield in soilless culture. J. Plant Dis. Protec. 106, 568-580.

Grosch, R., Koch, T., and Kofoet, A. (2004). Control of bottom rot on lettuce caused by Rhizoctonia solani with commercial biocontrol agents and a novel fungicide. J. Plant Dis. Protec. 111, 572-582.

Hartmann, A., Schmid, M., Van Tuinen, D., and Berg, G. (2009). Plant-driven selection of microbes. Plant Soil 321, 235-257. doi: 10.1007/s11104-008-9814-y 
Idriss, E. E., Bochow, H., Ross, H., and Borriss, R. (2004). Use of Bacillus subtilis as biocontrol agent. VI. Phytohormone-like action of culture filtrates prepared from plant growth-promoting Bacillus amyloliquefaciens FZB24, FZB42, FZB45 and Bacillus subtilis FZB37. J. Plant Dis. Protec. 111, 583-397.

Inceoglu, Ö., van Overbeek, L. S., Falcão Salles, J., and van Elsas, J. D. (2013). Normal operating range of bacterial communities in soil used for potato cropping. Appl. Environ. Microbiol. 79, 1160-1170. doi: 10.1128/AEM.02811-12

Johri, B. N., Sharma, A., and Virdi, J. S. (2003). "Fluorescent pseudomonads in plant disease management," in Biotechnological Approaches in Soil Microorganisms for Sustainable Crop Production, ed K. R. Dadarwal (Jodhpur: Scientific Publishers), 193-223.

Kazempour, M. N. (2004). Biological control of Rhizoctonia solani, the causal agent of rice sheath blight by antagonistics bacteria in greenhouse and field conditions. Plant Pathol. J. 3, 88-96. doi: 10.3923/ppj.2004.88.96

Koumoutsi, A., Chen, X.-H., Henne, A., Liesegang, H., Hitzeroth, G., Franke, P., et al. (2004). Structural and functional characterization of gene clusters directing nonribosomal synthesis of bioactive cyclic lipopeptides in Bacillus amyloliquefaciens strain FZB42. J. Bacteriol. 186, 1084-1096. doi: 10.1128/JB.186.4.1084-1096.2004

Koumoutsi, A., Chen, X.-H., Vater, J., and Borriss, R. (2007). DegU and YczE positively regulate the synthesis of bacillomycin D by Bacillus amyloliquefaciens strain FZB42. Appl. Environ. Microbiol. 73, 6953-6964. doi: 10.1128/AEM.00565-07

Krzywinski, M., Schein, J., Birol, I., Connors, J., Gascoyne, R., Horsman, D., et al. (2009). Circos: an information aesthetic for comparative genomics. Genome Res. 19, 1639-1645. doi: 10.1101/gr.092759.109

Leistra, M., and Matser, A. M. (2004). Adsorption, transformation, and bioavailability of the fungicides carbendazim and iprodione in soil, alone and in combination. J. Environ. Sci. Health B 39, 1-17. doi: 10.1081/PFC-120027435

Lugtenberg, B., and Kamilova, F. (2009). Plant-growth-promoting rhizobacteria. Annu Rev. Microbiol. 63, 541-556. doi: 10.1146/annurev. micro.62.081307.162918

Meyer, F., Paarmann, D., Souza, M., Olson, R., Glass, E., Kubal, M., et al. (2008). The metagenomics RAST server - a public resource for the automatic phylogenetic and functional analysis of metagenomes. BMC Bioinformatics 9:386. doi: 10.1186/1471-2105-9-386

Molassiotis, A., and Fotopoulos, V. (2011). Oxidative and nitrosative signaling in plants: two branches in the same tree? Plant Signal. Behav. 6, 210-214. doi: 10.4161/psb.6.2.14878

Oerke, E.-C. (2006). Crop losses to pests. J. Agric. Sci. 144, 31-43. doi: $10.1017 /$ S0021859605005708

Overbeek, R., Olsen, R., Pusch, G., Olsen, G., Davis, J., Disz, T., et al. (2014). The SEED and the rapid annotation of microbial genomes using subsystems technology (RAST). Nucleic Acids Res. 42, D206-D214. doi: 10.1093/nar/gkt1226

Pisa, G., Magnani, G., Weber, H., Souza, E., Faoro, H., Monteiro, R., et al. (2011). Diversity of 16S rRNA genes from bacteria of sugarcane rhizosphere soil. Braz. J. Med. Biol. Res. 44, 1215-1221. doi: 10.1590/S0100-879X2011007500148

Scherwinski, K., Grosch, R., and Berg, G. (2008). Effect of bacterial antagonists on lettuce: active biocontrol of Rhizoctonia solani and negligible, short-term effects on nontarget microorganisms. FEMS Microbiol. Ecol. 64, 106-116. doi: 10.1111/j.1574-6941.2007.00421.x

Steidle, A., Allesen-Holm, M., Riedel, K., Berg, G., Givskov, M., Molin, S., et al. (2002). Identification and characterization of an $\mathrm{N}$-acylhomoserine lactone-dependent quorum-sensing system in Pseudomonas putida strain IsoF. Appl. Environ. Microbiol. 68, 6371-6382. doi: 10.1128/AEM.68.12. 6371-6382.2002

Tiago, I., Teixeira, I., Silva, S., Chung, P., Verissimo, A., and Manaia, C. M. (2004). Metabolic and genetic diversity of mesophilic and thermophilic bacteria isolated from composted municipal sludge on poly-epsilon-caprolactones. Curr. Microbiol. 49, 407-414. doi: 10.1007/s00284-004-4353-0

Unno, Y., and Shinano, T. (2013). Metagenomic analysis of the rhizosphere soil microbiome with respect to phytic acid utilization. Microbes Environ. 28, 120-127. doi: 10.1264/jsme2.ME12181

Vasileiadis, S., Puglisi, E., Arena, M., Cappa, F., and Cocconcelli, P. (2012). Soil bacterial diversity screening using single $16 \mathrm{~S}$ rRNA gene $\mathrm{V}$ regions coupled with multi-million read generating sequencing technologies. PLOS ONE 7:e42671. doi: 10.1371/journal.pone.0042671

Wang, Y. S., Wen, C. Y., Chiu, T. C., and Yen, J. H. (2004). Effect of fungicide iprodione on soil bacterial community. Ecotoxicol. Environ. Saf. 59, 127-132. doi: 10.1016/j.ecoenv.2004.01.008

Wilke, A., Glass, E., Bischof, J., Braithwaite, D., Souza, M., Gerlach, W., et al. (2013). MG-RAST Technical Report and Manual for Version 3.3.6-Rev. 1.

Winding, A., Binnerup, S. J., and Pritchard, H. (2004). Non-target effects of bacterial biological control agents suppressing root pathogenic fungi. FEMS Microbiol. Ecol. 47, 129-141. doi: 10.1016/S0168-6496(03)00261-7

Conflict of Interest Statement: This work was part of the PathControl cooperative project 'Biological control of Bacillus amyloliquefaciens on the soil-borne fungal pathogen R. solani (FKZ 0315654B) funded by the BMBF and no conflict of interest needs to be declared. The authors declare that the research was conducted in the absence of any commercial or financial relationships that could be construed as a potential conflict of interest.

Received: 06 November 2013; accepted: 07 May 2014; published online: 27 May 2014. Citation: Kröber M, Wibberg D, Grosch R, Eikmeyer F, Verwaaijen B, Chowdhury SP, Hartmann A, Pühler A and Schlüter A (2014) Effect of the strain Bacillus amyloliquefaciens FZB42 on the microbial community in the rhizosphere of lettuce under field conditions analyzed by whole metagenome sequencing. Front. Microbiol. 5:252. doi: 10.3389/fmicb.2014.00252

This article was submitted to Plant-Microbe Interaction, a section of the journal Frontiers in Microbiology.

Copyright (c) 2014 Kröber, Wibberg, Grosch, Eikmeyer, Verwaaijen, Chowdhury, Hartmann, Pühler and Schlüter. This is an open-access article distributed under the terms of the Creative Commons Attribution License (CC BY). The use, distribution or reproduction in other forums is permitted, provided the original author(s) or licensor are credited and that the original publication in this journal is cited, in accordance with accepted academic practice. No use, distribution or reproduction is permitted which does not comply with these terms. 